\title{
Stable Colloidal Quantum Dot Inks Enable Inkjet-Printed High-Sensitivity Infrared Photodetectors
}

\author{
Rafal Sliz †, Marc Lejay $\dagger$, James Z. Fan $\dagger$, Min-Jae Choi †, Sachin Kinge §, Sjoerd Hoogland †, Tapio \\ Fabritius $\ddagger$, F. Pelayo García de Arquer †, Edward H. Sargent $* \dagger$ \\ $\dagger$ Department of Electrical and Computing Engineering, University of Toronto, 10 King's College Road, Toronto, Ontario \\ M5S 3G4, Canada \\ \$ Optoelectronics and Measurement Techniques Unit, University of Oulu, 90570 Oulu, Finland \\ $\S$ Advanced Technology Materials and Research, Research and Development, Toyota Motor Europe, Hoge Wei 33, Toyota \\ Technical Centre, B-1930 Zaventem, Belgium
}

\begin{abstract}
Colloidal Quantum Dots (CQDs) have recently gained a lot of attention as a potential material for manufacturing optoelectronic devices due to their tunable light absorption and emission properties and compatibility with low-temperature thin-film manufacturing. The realization of CQD inkjet-printable infrared photodetectors has thus far been hindered by the incompatibility between the chemical processes that produce state-of-the-art CQD solution-exchanged inks and the inkjet material processing. To achieve inkjet-printed CQD solids with excellent reproducibility, morphology and optoelectronic characteristics, the discrepancy between these processing conditions needs to be reconciled. In this study, we design CQD inks by simultaneous evaluation of requirements regarding ink colloidal stability, jetting conditions and the film morphology for different dots and solvents. Our inks remain colloidally stable through a design that suppresses the reductant properties of amines on the dots' surface. After drop ejection from the nozzle, the quantum dot material is immobilized on the substrate surface due to the rapid evaporation of the low boiling point amine-based compound. Concurrently, the high boiling point solvent allows for the formation of a thin film of high uniformity, which is required for the fabrication of high-performance IR photodetectors. We then fabricate inkjet-printed photodetectors exhibiting the highest specific detectivities reported to date (above $10^{12}$ Jones across the IR) in an inkjet-printed film. As a patternable CMOS-compatible process, the work offers routes to integrated sensing devices and systems.
\end{abstract}

\section{KEYWORDS}

CQD, colloidal quantum dots, inkjet, ink-jet, printing, photodetectors, infrared 
CQDs are emerging optoelectronic nanomaterials with tunable absorption and emission properties that are also compatible with low-temperature thin-film manufacturing. ${ }^{1,2}$ The synthesis and processing of CQDs have progressed significantly in recent years, resulting in the implementation of efficient optoelectronic devices such as bright CQD LEDs, ${ }^{3-5}$ flexible transistors, ${ }^{6}$ solar cells with power conversion efficiencies exceeding $12 \%,{ }^{7,8}$ and high-efficiency infrared photodetectors. ${ }^{9,10}$ These advances have stemmed from improvements in CQD synthesis, surface passivation, and device architecture. ${ }^{11-17}$

Ligand exchanges are essential to realizing electrically-conductive CQD solids since in this step, the original insulating ligands are replaced with shorter, conduction-compatible ones and surface defects are minimized (Figure 1a). ${ }^{18,19}$ There are two approaches in ligand-exchange protocols: solid-state layerby-layer (LbL) and solution-phase. In the LbL method, original ligands are replaced sequentially during film growth by ligand dosing and solvent rinsing. In solution-phase ligand exchanges, short ligands or anions replace the original longer ligands while the dots are colloidally dispersed. These CQD inks can be directly deposited to the substrate, enabling single-step fabrication of CQD devices. ${ }^{20}$ In recent studies, $\mathrm{CQD}$ inks based on lead halide $\left(\mathrm{PbX}_{2}\right)$ anions have yielded the best-performing photovoltaics owing to the homogeneous energy landscape provided by the resultant CQD solids. ${ }^{7}$ The homogeneous energy landscape narrows the distribution of electronic states at the band edges, reducing the amount of bandtail states, resulting in improved open-circuit voltage in solar cells and photodetectors. However, the $\mathrm{PbX}_{2}-$ exchanged CQD inks remain as colloids only in volatile amine solvents (i.e. $n$-butylamine), ${ }^{21}$ which cause surface etching and degradation of the CQDs (Figure 1b). In addition, their low boiling point and viscosity make these formulations inapplicable to large-scale manufacturing processes such as inkjet printing and screen-printing. ${ }^{14}$

The realization of CQD inks that achieve excellent passivation while retaining colloidal stability is required for successful demonstration of inkjet printing (Figure 1c). In addition, ink rheological properties such as viscosity, surface tension, boiling point, particle size, and concentration must be carefully controlled to enable optimal inkjet performance, without introducing constraints over CQD surface passivation or subsequent self-assembly that negatively affect the optoelectronic properties of the printed films. ${ }^{8}$ Various solvents and their mixes provide the ability to control printing properties but these often fail to balance the attractive and repulsive forces in colloids, resulting in a decrease of solubility or loss of excitonic peak (Figure 1d). 
a

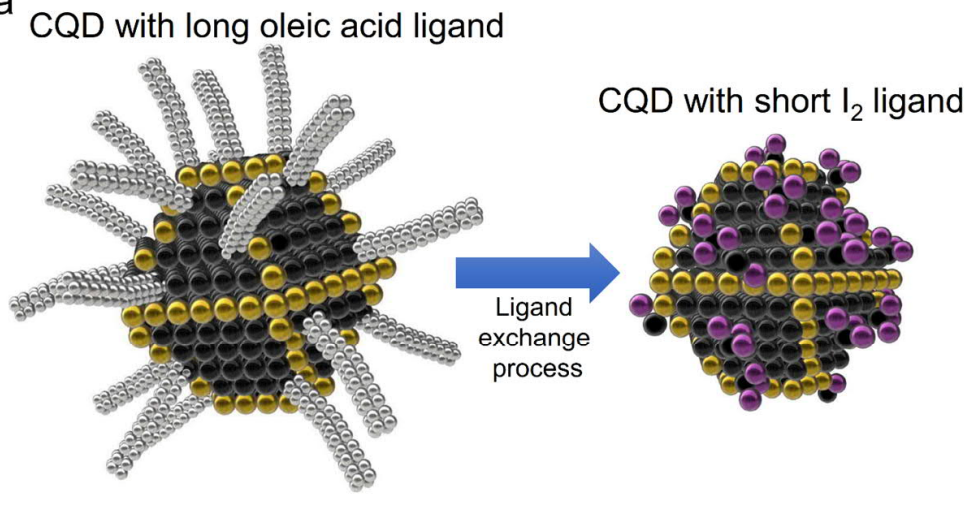

b

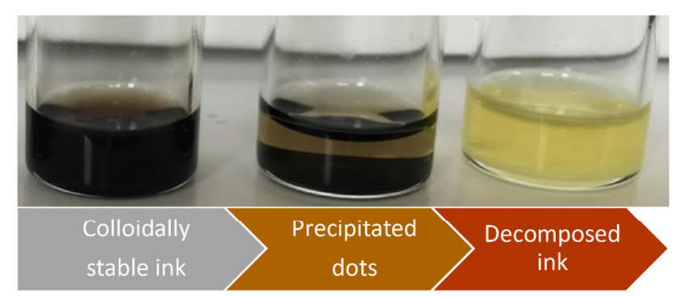

d

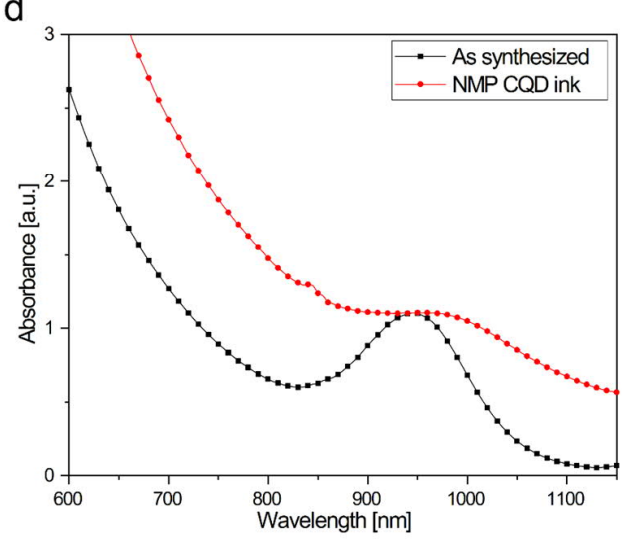

Figure 1. Solution-exchanged CQD inks and inkjet printing-related concerns. a) Ligand-exchange process where the long oleic acid ligand is replaced with shorter $I_{2}$ ligand. This process improves the charge transfer properties of fabricated thin films. b) $n$-Butylamine-based ink and related quantum dots etching degradation stages: Left: good quality quantum dot ink, exhibiting adequate redispersion and minimal dot aggregation. Middle: ink in which most quantum dots have quickly precipitated from solution, impeding the printing process. Right: solution of fully etched quantum dots. Decomposition back into precursors causes loss of absorptive properties. The decomposition occurs in a range of hours. c) Inkjet printing requires stable CQD inks, achieved in two ways: Electrostatic stabilization (1): charges on the dot surface attract oppositely charged ions in the solvent to create a buffer layer. Repulsion between these layers, which is contingent on shielding the original particle charge, keeps particles apart. Solvent dielectric constant strength is crucial in determining the level of charge shielding. Steric stabilization (2): given proper solvent selection based on polarity, the mixing of ligands becomes energetically unfavorable. This prevents dot aggregation and imparts colloidal stability. d) Absorbance spectra of $950 \mathrm{~nm}$ quantum dots after synthesis and after ligand-exchange redispersed in NMP solvent. Although NMP allows ink-jet printing, it diminishes the quantum dot exciton behavior. 
Our efforts concentrate on inkjet printing which belongs to a drop-on-demand technology that allows dispensing and accurate positioning of small droplets of material-consisting fluid on a substrate, resulting in desired patterns and structures. This is essential to create optoelectronic devices with different functionalities that can be monolithically integrated and to reduce material waste during their fabrication. Throughout the inkjet printing process, picoliter drops of ink are ejected from a nozzle by applying a pulse of pressure to the fluid ink in the supply tube. Nozzles used for inkjet printing have sizes ranging from 10 to $150 \mu \mathrm{m},{ }^{22,23}$ requiring highly colloidally stable inks. Unstable inks frequently cause nozzle clogging or negatively affect the printed thin film quality and consequently reduce the optoelectronic performance of the final devices.

Many researchers have investigated the mechanisms of drop formation with the aim of providing guidelines for optimal ink formulation. ${ }^{24-26}$ Nonetheless, the large number of relevant parameters impedes the development of a universal guideline for optimal ink formulation. An interesting approach has been proposed by Nallan et al. in which several parameters affecting inkjet drop formation have been considered, leading to the concept of jettability window guidelines. ${ }^{27}$ Complications regarding ink formulation are further compounded when printing full device stacks, where factors like the interactions and compatibility of the fabricated inks with layers beneath must be taken into account. Although inkjet printing is a promising fabrication method, only a limited number of researchers have to date inkjetprinted CQD IR photodetectors, mostly due to difficulties with colloidal stability and printability of the CQD inks and consequently reduced performance of the final devices. ${ }^{28,29}$ Table 1 provides insight into recent advancements in printed/solution processed CQD photodetectors.

Table 1: Comparison of various parameters of printed photodetectors. Works of Böberl et al. and Tang et al. represent printed or otherwise patterned detectors. The remaining references stand for solution-processed photodetectors, deposited by other methods. The high detectivity of the inkjet-printed devices presented in this work makes them stand out among patterned IR detectors. Their large size and promising efficiency make them competitive with detectors produced through more conventional solution-process methods.

\begin{tabular}{|c|c|c|c|c|c|c|}
\hline Reference & Structure & $\begin{array}{c}\text { Area } \\
\left(\mathrm{mm}^{2}\right)\end{array}$ & $\begin{array}{l}\mathrm{EQE} \\
(\%)\end{array}$ & $\begin{array}{c}\mathrm{D}^{*} \\
\text { (Jones) }\end{array}$ & $\begin{array}{l}\text { Range } \\
(\mathrm{nm})\end{array}$ & Note \\
\hline Böberl 200729 & Ti/Au:Hg Te QD & 10 & 53 & $3.2 \times 10^{10}$ & $500-3000$ & Printed PC \\
\hline Tang $2016^{30}$ & $\mathrm{Cr} / \mathrm{Au}: \mathrm{Hg} \mathrm{Te} \mathrm{QD}$ & - & 6.2 & - & $2000-8000$ & PMMA transfer \\
\hline Clifford $2008^{9}$ & $\mathrm{PbS}$ QD & - & 17 & $1 \times 10^{12}$ & $400-1800$ & Schottky \\
\hline Pal $2012^{31}$ & Pedot:PbS QD:ZnO & 9 & 20 & $1.1 \times 10^{12}$ & $300-1100$ & PN PD \\
\hline Manders $2014^{32}$ & NiO:PbS QD:ZnO & 4.5 & 24 & $1.1 \times 10^{12}$ & $400-1300$ & All inorganic \\
\hline Masala $2015^{33}$ & c-Si:PbS QD:AZO & 4.9 & 33 & $1.5 \times 10^{11^{*}}$ & $400-1200$ & Si heterojunction \\
\hline Kim $2015^{34}$ & $\mathrm{TiO}_{2}: \mathrm{PbS} \mathrm{QD}$ & - & 39 & $2.4 \times 10^{13}$ & $400-1100$ & Quantum funnel \\
\hline This work & ZnO:PbS QD:Spiro & 11.5 & $>40$ & $>10^{12}$ & $400-1650$ & Inkjet-printed \\
\hline
\end{tabular}




\section{RESULTS AND DISCUSSION}

\section{Ink colloidal stability and printability}

The CQD ligand exchange was performed following the procedure developed by Liu et al. ${ }^{7}$ After drying, the dots were redispersed in various solvents and their stability and suitability for inkjet printing analyzed. To achieve colloidally stable, printable functional CQD inks, we examined three promising solvents: $n$-butylamine (BTA), dimethylformamide (DMF), and N-Methyl-2-Pyrrolidone (NMP).

The solvent selection was based on their potential to redisperse and stabilize dots, inkjet printability, and ability to form smooth thin films. At this stage, we simultaneously evaluated the requirements regarding ink colloidal stability and jetting conditions by measuring the main parameters of the fabricated inks (viscosity, density, surface tension, nozzle diameter, and droplet velocity) and their fit to the ink printability window depicted in Figure 2a (details of the measurements and calculations are attached in the Supporting Information).

In CQD research, BTA is commonly used as a solvent because it easily redisperses the dots. However, it is also known to act as a reducing agent, causing dot precipitation and surface etching (Figure 1b). This compromises the stability of the solution required by the inkjet printing process. In addition, the very low boiling point $\left(78^{\circ} \mathrm{C}\right)$ and low viscosity $(500 \mu \mathrm{Pa} \cdot \mathrm{s})$ make it unsuitable for inkjet printing because rapid evaporation at the nozzle causes a build-up of particles that interfere with the jetting process. While the remaining inkjet printing parameters indicate its potential for printability (Figure 2a), the low boiling point and dot precipitation disqualify printing and therefore BTA was excluded from further consideration.

DMF, another common solvent, has a demonstrated ability to stabilize the dots, adequate printing parameters and a preferable boiling point of $153{ }^{\circ} \mathrm{C} .{ }^{7}$ Nevertheless, the consistent generation of drops with various inkjet printers was challenging and therefore the solvent was considered unsuitable.

A high boiling point of $202{ }^{\circ} \mathrm{C}$ and promising inkjet printing parameters (viscosity, density, and surface tension) make NMP a good co-solvent for inkjet printing of CQDs. ${ }^{35}$ Importantly, the polarity (6.7) and dielectric constant (32.2) of NMP are similar to those of DMF (6.4 and 36.7, respectively), enabling it to keep the exchanged dots stably redispersed. Despite the similarity of its properties to DMF, NMP does not fully redisperse the dots, resulting in washed-out exciton peaks normally attributed to quality CQD solutions (Figure 1d). However, since NMP provided excellent printability and allowed stable jetting of the CQD ink, we considered it to be a prime solvent used to print several reference thin 
films in order to optimize the printing process, printing speed, droplet volume, and substrate compatibility.

Based on the previous experiments, we focused our efforts on obtaining quality inks by taking advantage of the high CQD solubility in BTA and the high printability of NMP. Taking into account the possibility that BTA might act as a ligand, we tested only relatively small ratios of BTA to NMP. ${ }^{12}$ After experimenting with various compositions of BTA to NMP, we recognized that the most optimal ink consists of NMP with the addition of $1 \mathrm{wt} \%$ BTA (BTA:NMP).

The low ratio of BTA to NMP suppressed the reductive influence of BTA resulting in a colloidally stable and printable solution (after several days the ink remains without any indicators of aggregation), as seen in Figure 2b. BTA to NMP ratios higher than $1 \mathrm{wt} \%$ did not significantly improve redispersity but did reduce the colloidal stability of the inks. Also, higher ratios of BTA compromised the printability by clogging the nozzles. At the same time, pure NMP ink without BTA, depicted in Figure $2 b$, exhibited visible CQD agglomerations. Figures $2 \mathrm{c}, \mathrm{d}$ illustrate the ejection and droplet creation process of the BTA:NMP CQD ink for two different printers, demonstrating interoperability of the created ink. By using one printing nozzle, it takes approximately ten minutes to print $11.5 \mathrm{~mm}^{2}$ of the active layer but current industrial inkjet printers have the capacity to print such structures in seconds. 
a

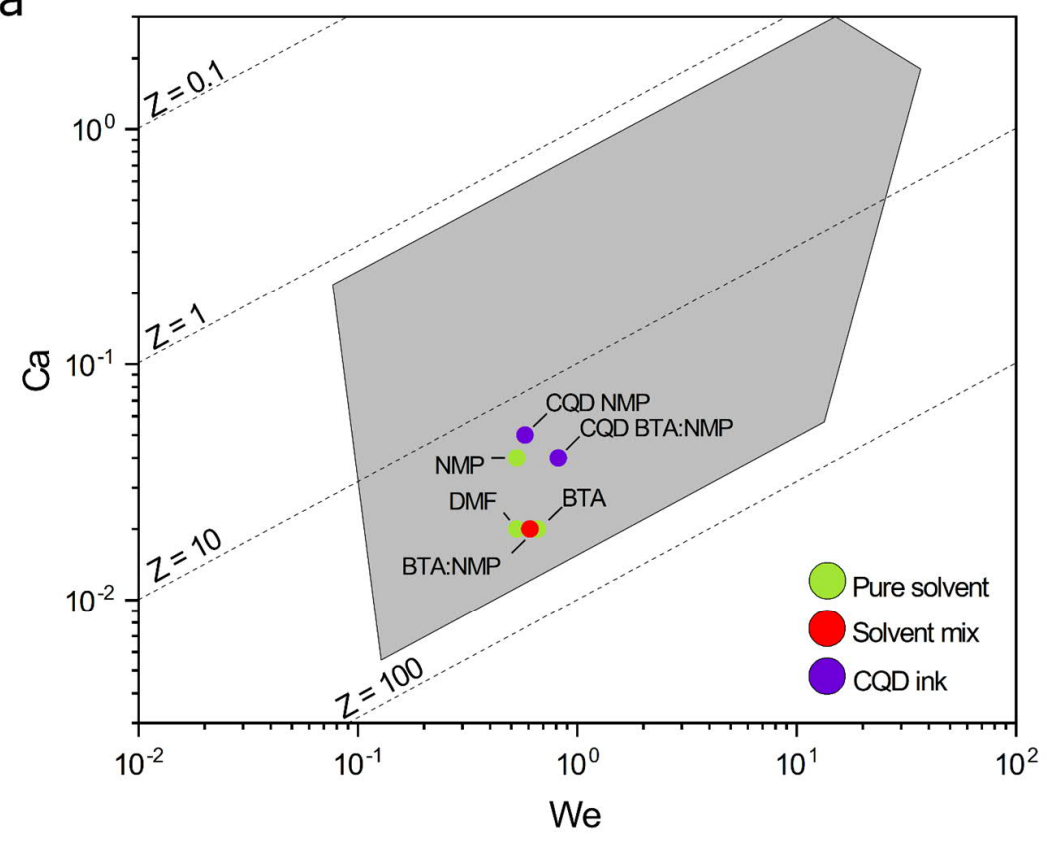

C

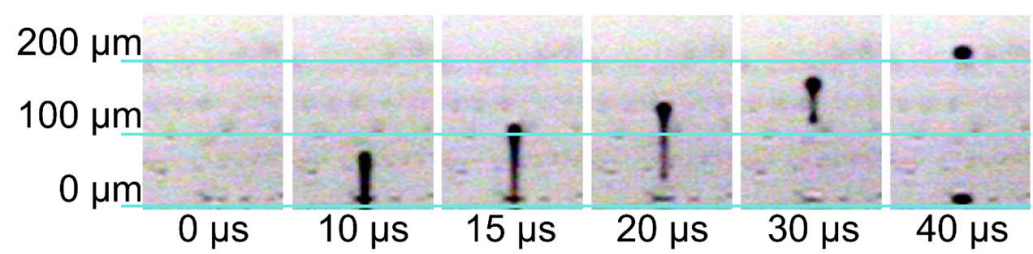

b

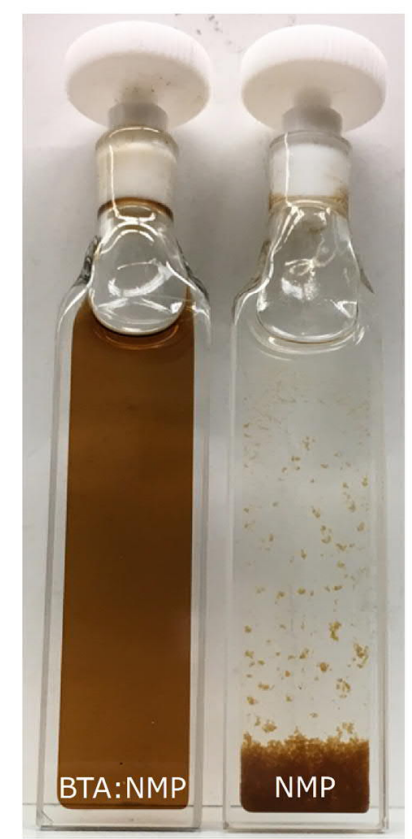

d

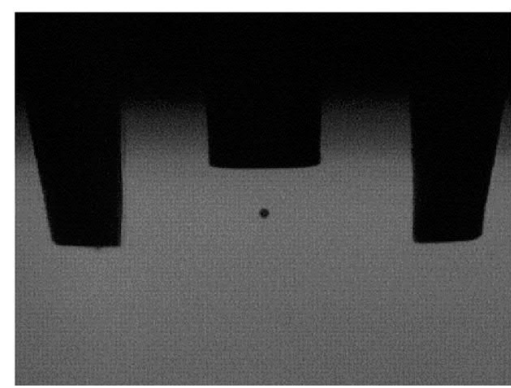

Figure 2. Analysis of jettability/printability and colloidal stability of the CQD inks. a) Jettability window within the Capillary and Weber number (Ca-We) space proposed by Nallan et al. ${ }^{27}$ All inks and solvent that fit within the window should allow the creation of stable drops. The Weber number is a dimensionless number used as a measure of the relative importance of the fluid's inertia compared to its surface tension. The Capillary number represents the relative effect of viscous drag forces versus surface tension forces acting across an interface between a liquid and a gas. Colors indicate the ink composition: green - pure solvent, red - solvent mix, violet - CQD ink. Although all formulated and tested inks lay within the window, BTA ink does not allow jetting due to its low boiling point and the nozzle clogging. b) Comparison of two inks - BTA:NMP and NMP-only. The addition of BTA creates a well redispersed and stable colloidal solution while the NMP-only ink demonstrates signs of aggregation. c) Demonstration of BTA:NMP drop formation for Fujifilm Dimatix DMP-2800 10 pl printing head. The drop is fully formed after $40 \mu \mathrm{s}$ from ejection and at $200 \mu \mathrm{m}$ from the nozzle. Additional video demonstrating stable BTA:NMP drop formation for 6 parallel nozzles is provided in the Supporting Information. d) Creation of stable droplet of BTA:NMP ink in MicroFab Jetlab 4 XL printing nozzle. 


\section{CQD thin films formation and characterization}

After determining an optimal composition, the BTA:NMP ink was used to print CQD thin films for morphological and IR photodetector characterization. To account for the influence of substrate surface on film morphology, all prints were done on Zinc Oxide $(\mathrm{ZnO})$ thin films. These films would ultimately be used as an electron transport layer for (PIN architecture) IR photodetectors.

Optical and atomic force microscopy images shown in Figure 3a,b,c compare the printed CQD thin films resulting from different inks. Aggregations of CQDs in the NMP-only ink are transferred to the film, yielding high roughness, as indicated in the AFM results (RMS $44.7 \mathrm{~nm}$ ) (Figure 3a). On the other hand, the BTA:NMP ink provides excellent morphological performance - smooth layers with very low roughness (RMS $3.3 \mathrm{~nm}$ ) (Figure 3b). The films were optimized further by elevating the substrate temperature, which promotes faster solvent evaporation and material pinning processes that immobilize the solid content, resulting in more uniform films. Microscope and AFM images demonstrate that the roughness of CQD thin films printed at $60{ }^{\circ} \mathrm{C}$ is reduced to RMS $3.0 \mathrm{~nm}$ and the material distribution at the macroscopic scale is more even (Figure 3c).

To better understand the drying process of the inks on the substrate, we supplemented optical and atomic force microscopy examination with thermogravimetric (TGA) analysis. The results are shown in Figure 3d. For BTA:NMP inks, the TGA results reveal that the drying process (losing weight) starts at significantly lower temperatures thanks to the BTA component that evaporates rapidly, thus immobilizing the CQD material, while the high boiling point solvent (NMP) evaporates steadily allowing the material to settle and create a continuous, smooth layer. 

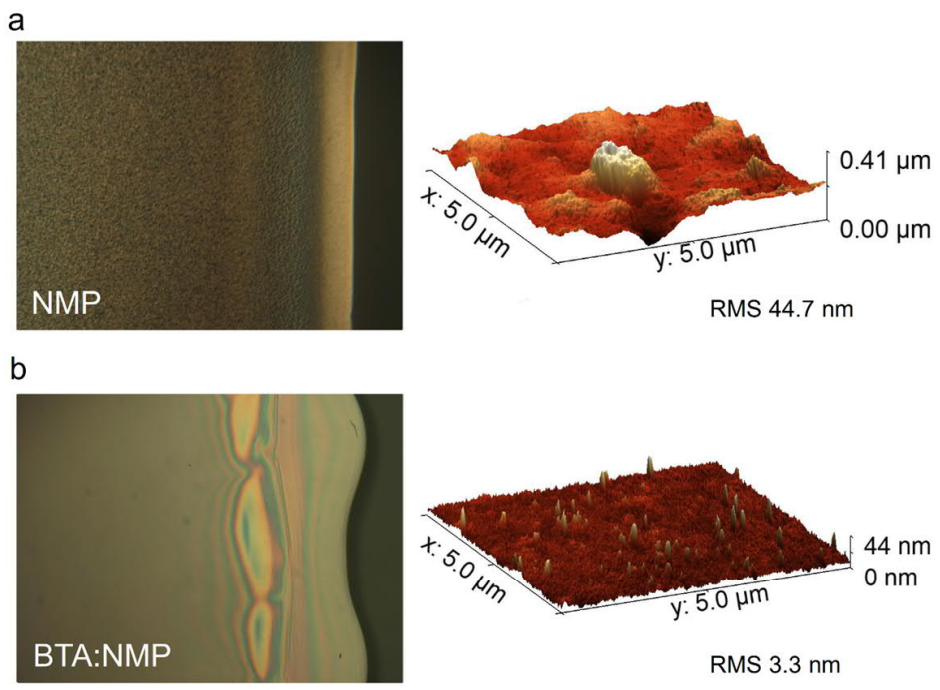

C
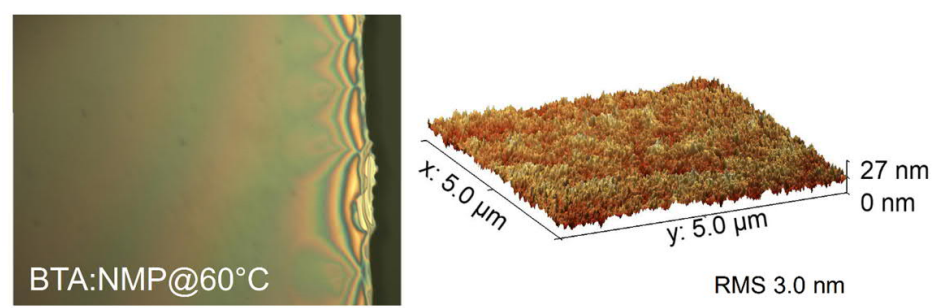

d

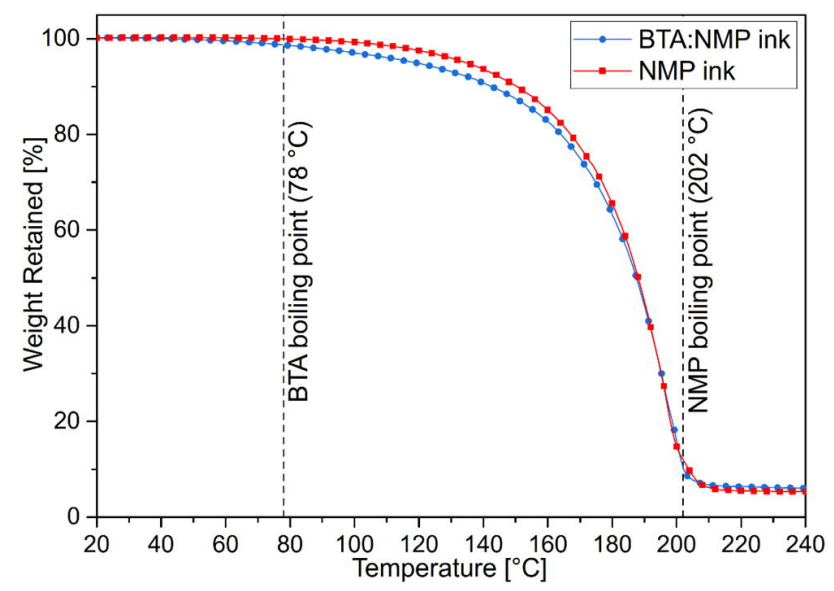

Figure 3. CQD thin film morphology and ink drying process. a) Microscope and AFM analysis of a film printed with NMP-only ink. The RMS value has been calculated from the 5x5 $\mu \mathrm{m}$ area. b) Microscope and AFM analysis of a film printed with BTA:NMP ink. Compared to films made with NMP-only ink, the RMS roughness is significantly reduced, from $44.7 \mathrm{~nm}$ to $3.3 \mathrm{~nm}$. c) Microscope and AFM analysis of a film printed with BTA:NMP ink on a substrate heated to $60{ }^{\circ} \mathrm{C}$. The roughness has been further reduced and the larger scale uniformity improved. d) Thermogravimetric analysis (Netzsch STA 449 F3 Jupiter) of BTA:NMP and NMP inks. Compared to NMP ink, the BTA component in the BTA:NMP ink induces drying at significantly lower temperatures. After drying, both inks retain approximately $5 \%$ of the initial material weight. 
Both types of inkjet-printed thin films (BTA:NMP and NMP-only) underwent elemental analysis using X-ray photoelectron spectroscopy (XPS). The survey results indicate that the difference between BTA:NMP and NMP inkjet-printed films is insignificant (additional information is provided in the Supporting Information). Transmittance measurements of as-synthesized and printed thin films reveal that both as-synthesized and exchanged NMP:BTA dots exhibit exciton peaks, while the peak is diminished for the NMP-only ink (Figure S4). Since the NMP-only films did not demonstrate clear exciton peaks, we concentrated our photodetector-fabrication efforts on the BTA:NMP inks.

\section{Fabrication and characterization of IR photodetectors}

$\mathrm{ZnO}$ has successfully been used as a solution-processed carrier extracting material in $\mathrm{PbS} \mathrm{QD}$ based photodiodes and efficient solar cells. ${ }^{36} \mathrm{We}$, therefore, selected it as the $n$-type layer in our PIN photodetectors.

To enable eventual printing of all layers in the photodetector, we limited the selection of the $p$ type material to solution-processed materials (Figure 4a). The key requirement for this layer was to allow efficient extraction of holes from the quantum dot film. The $p$-type material was also required to keep the dark current of the photodetector as low as possible to improve photodetector performance. ${ }^{37}$

Inspired by the rapid advances in perovskite optoelectronics, we tested an organic material, Lithium-doped 2,2',7,7'-tetrakis(N,N-di-4-methoxyphenylamino)-9,9'-spirobifluorene (Spiro), as the $p$ type layer ${ }^{38}$ Spiro is a commonly used Hole Transport Layer (HTL) in perovskite solar cells, which have reached power conversion efficiencies of $23.3 \%$. It is an excellent candidate for a HTL because the valence band aligns well with Au for hole extraction and its wide bandgap blocks electron back-injection (Figure $4 b$ ). ${ }^{33}$ As a first step, film thicknesses were optimized given that Spiro has a much lower hole mobility than the electron mobility of $\mathrm{ZnO}\left(1.6 \times 10^{-3}\right.$ vs. $\left.0.5 \mathrm{~cm}^{2} / \mathrm{V} \cdot \mathrm{s}\right) \cdot{ }^{39,40}$ Multiple other factors also contribute to thickness-dependent performance. One such factor is the absorption-extraction compromise, wherein photon absorption and carrier extraction efficiencies must be balanced..$^{41}$ Reverse biasing can overcome this requirement by enhancing the internal field, but this technique can only be used if the film can sustain high electric fields without breakdown or significant leakage. Another factor is the creation of Fabry-Perot resonances. Variations in layer thicknesses can tune the locations of these resonances and potentially enhance efficiency at the exciton. Color-enhanced SEM imaging shows the final, thickness-optimized photodetector stack (Figure 4c). 
The performance of the inkjet-printed photodetectors was analyzed through several key metrics: dark J-V curve, reverse-bias dependent efficiency, and detectivity. Details of the measurement protocols, the set-ups used and additional characterization results (photodetector bandwidth, irradiance-dependent responsivity, and noise equivalent power (NEP)) are provided in the Supporting Information.

$\mathrm{J}-\mathrm{V}$ measurements were performed by sweeping the bias voltage from $-1 \mathrm{~V}$ to $1 \mathrm{~V}$ and measuring the diode response. Results are shown in Figure 4d. Standard EQE scans taken at various bias voltages determine the reverse-bias dependent efficiency (Figure 4e). Overall upward shifts to otherwise similar efficiency spectra indicate better photocurrent generation and extraction. The comparable efficiencies at shorter wavelengths of samples biased at $0.5 \mathrm{~V}$ and $1 \mathrm{~V}$ indicate that carrier extraction saturates. EQEs are converted to detectivities, which show consistent device performance across its spectral range with a $\mathrm{D}^{*}$ of $1.4 \times 10^{12}$ Jones (Figure $4 \mathrm{f}$ ). For comparison, peak reported values of $\mathrm{D}^{*}$ in commercial Ge and InGaAs detectors are on the order of 0.35 and $6 \times 10^{12}$ Jones, respectively. ${ }^{32}$ Initial trials of printed devices with larger size dots (1250 and $1500 \mathrm{~nm}$ ) show that similar performance can be achieved further in the infrared using the same process developed herein. 
a
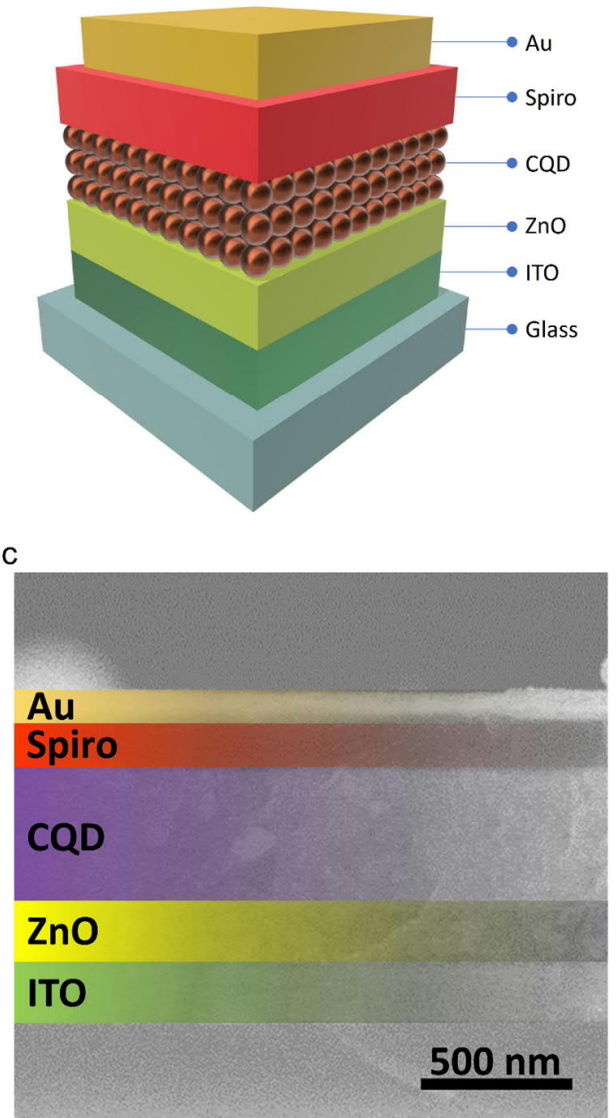

e

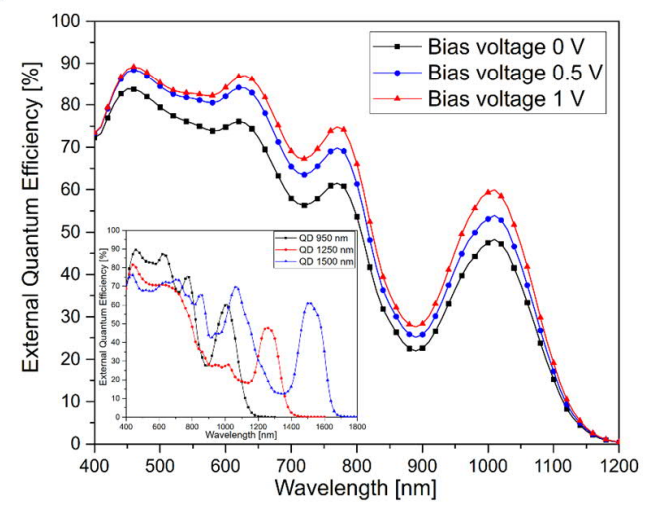

b

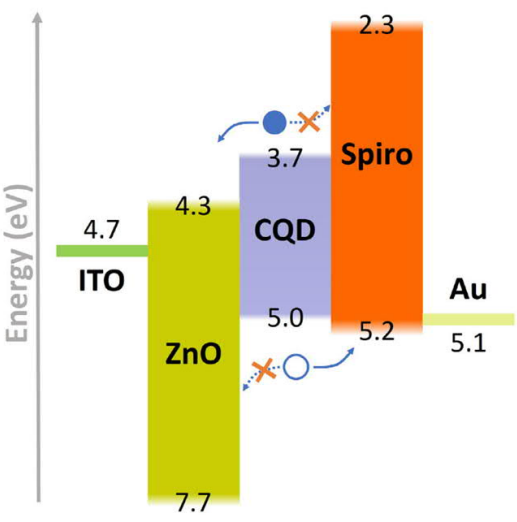

d

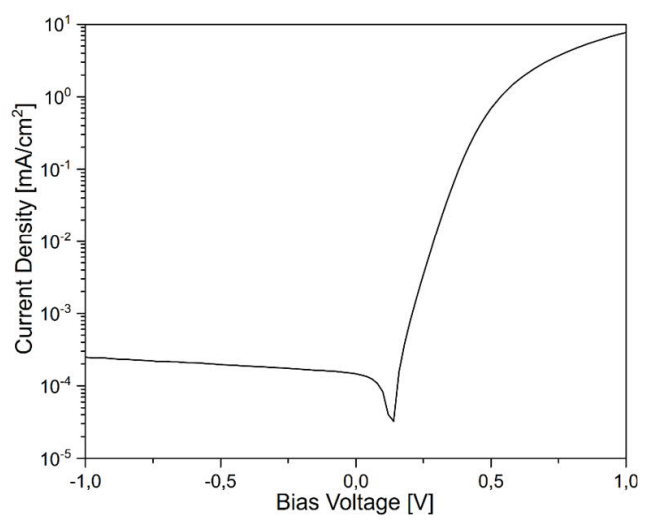

f

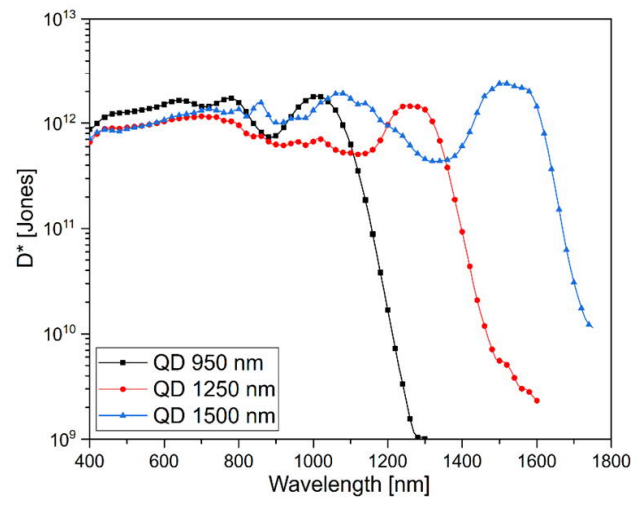

Figure 4. Photodetector architecture and characterization. a) IR photodetector architecture with ITO as the transparent conductive electrode, $\mathrm{ZnO}$ as the electron transport layer, $\mathrm{CQDs}$ as the active layer, Spiro as the hole transport layer, and gold as the top electrode. b) Energy band alignment of the final CQD IR photodetector. The image demonstrates how Spiro helps to enhance electron extraction. c) SEM image representing the full photodetector stack. ITO and ZnO thicknesses are approximately $150 \mathrm{~nm}$. The printed CQD layer is approximately $350 \mathrm{~nm}$ thick and Spiro thickness is approximately $150 \mathrm{~nm}$. E-beam-deposited gold electrode is approximately $100 \mathrm{~nm}$. d) Dark J-V curve of the ink-jet printed photodetector demonstrating anticipated diode behavior. e) Biasdependent EQE of printed CQD photodiodes. Reverse-bias widens the depletion region and enhances photocurrent 
generation. Insert demonstrates EQE of printed CQD photodetectors with different size quantum dots $(950 \mathrm{~nm}$, $1250 \mathrm{~nm}$, and $1500 \mathrm{~nm}$ ) with $1 \mathrm{~V}$ bias. f) Detectivity of printed CQD photodetectors with different size quantum dots $(950 \mathrm{~nm}, 1250 \mathrm{~nm}$, and $1500 \mathrm{~nm})$. Consistent detectivity of approximately $1.4 \times 10^{12}$ Jones is achieved across the device's spectrum, taken for a $10 \mathrm{~Hz}$ signal. Spectra were acquired using $1 \mathrm{~V}$ reverse bias. Larger sized dots can extend the device spectral sensitivity to sense light further in the IR with similar performance.

In this work, we developed efficient and sensitive $\mathrm{PbS}$ quantum dot photodetectors using inkjet printing as a deposition method. Building on recent successes in forming high-quality exchanged dot solutions, we established a protocol to create high-quality inkjet printable CQD inks. We resolved the difficulties related to incompatibility between solvent composition, colloidal stability, printability, and optoelectronic properties of the printed films by continuous and comprehensive evaluation of the ink rheology, jetting conditions and the morphology of the printed films. Although we recognized that all studied inks lay within the printability window of the Capillary number - Weber number space, not all of them have been suitable for CQD applications due to either nozzle clogging or CQD aggregation. Despite its limitations, the concept of the printability window provided a good starting point for the development of suitable ink formulations.

We utilized the best ink formulation to further experiment with device structures, ultimately achieving efficient, large-area photodetectors working in the visible and near-IR regions. Extended characterization of the devices revealed that they are competitive with other reported $\mathrm{PbS}$ devices made using lab-scale solution processing methods. In certain metrics, such as detectivity, our inkjet-printed devices achieve performance levels similar to those of conventional crystalline materials. With their proven ability to create promising photodetectors, the freedom in patterning of inkjet printing and detection range of $\mathrm{PbS}$ quantum dots combine to create an opportunity for innovation in the field of IRphotodetection.

Our findings suggest several courses of action for further research to fully realize the potential of printed IR CQD photodetectors. There is a need for quantification and engineering of Fabry-Perot resonances that could augment certain spectral features. Further research might also explore various HTL materials that could increase the speed and efficiency of the photodetectors by improving carrier transport. Another interesting consideration is to utilize our protocol to develop fully printed IR photodetectors. 


\section{CONCLUSIONS}

In this work, we realize a CQD ink that combines excellent colloidal stability with inkjet printing compatibility and anticipated thin film formation. We design inks that are colloidally stable within the jettability window without compromising CQD surface passivation. We then employ these inks to demonstrate inkjet-printed photodetectors with the highest specific detectivities (above $10^{12}$ Jones in the near IR) reported to date. This CMOS-compatible printing process allows the realization of integrated multiwavelength hyperspectral sensors.

\section{EXPERIMENTAL SECTION}

Quantum dot ligand exchange process

Oleic-acid-capped CQDs were synthesized using previously published methods. ${ }^{42}$ The $\mathrm{PbX}_{2} / \mathrm{AA}$ solution-phase ligand-exchange process was carried out in a test tube in air. Lead halides (lead iodide $0.1 \mathrm{M}$ and lead bromide $0.02 \mathrm{M})$ and $\mathrm{NH}_{4} \mathrm{Ac}(0.04 \mathrm{M})$ were pre-dissolved in dimethylformamide (DMF). $5 \mathrm{ml}$ of CQD octane solution $\left(10 \mathrm{mg} \mathrm{ml}^{-1}\right)$ was added to $5 \mathrm{ml}$ of the precursor solution. These were mixed vigorously for 1-2 min until the CQDs completely transferred to the DMF phase. The DMF solution was washed three times with octane. After ligand exchange, CQDs were precipitated via the addition of toluene and separated by centrifugation. After 10 min of drying in a nitrogen atmosphere, the dots were redispersed in the solvents $\left(100 \mathrm{mg} \mathrm{ml}^{-1}\right)$ and filtered $(0.2 \mu \mathrm{m}$ PTFE filter $)$ to facilitate the inkjet printing.

\section{Photodetector fabrication}

The ITO glass substrates were cleaned by sonication in acetone and isopropanol for $30 \mathrm{~min}$ each. After drying, the $\mathrm{ZnO}$ solution was spin-coated onto ITO glass at 3,000 r.p.m. for 20 s. The $\mathrm{ZnO}$ nanoparticles were synthesized following a published method. ${ }^{36}$ Consequently, a layer of PbS CQDs was inkjet printed. The inkjet-printed layer was then annealed on the heating plate for $5 \mathrm{~min}$ at $100{ }^{\circ} \mathrm{C}$. The annealing was followed by deposition of Spiro as described in Tan's report. ${ }^{43}$ The photodetector was finalized by Ebeam vacuum deposition of $120 \mathrm{~nm}$ of gold that serves as the top electrode. The photodetector area was $11.5 \mathrm{~mm}^{2}$. Additional description of printing, material properties, sample fabrication, and characterization can be found in the Supporting Information. 


\section{ASSOCIATED CONTENT}

\section{Supporting Information}

Supporting Information is available from the ACS Publications website or the authors. The files consist of the following information:

- Explanation of the jettability calculation as well as parameters of the investigated inks (surface tension, density, viscosity, $\mathrm{Re}, \mathrm{We}, \mathrm{Ca}$, and $\mathrm{Z}$ numbers) and printing conditions.

- Video demonstrating the CQD-ink drop ejection from 6 nozzles of the Dimatix DMP-2800 printer.

- XPS analysis of the fabricated thin films.

- Absorbance measurements of the fabricated films and synthesized CQDs.

- Detailed electrical characterization protocol and results, including bandwidth measurements, noise and detectivity, and irradiance dependent responsivity.

\section{AUTHOR INFORMATION}

Corresponding author: Edward H. Sargent, ted.sargent@utoronto.ca

\section{ORCID:}

Rafal Sliz: 0000-0002-7224-2426

Marc Lejay: 0000-0001-8562-7133

James Z. Fan: 0000-0002-1594-865X

Min-Jae Choi: 0000-0002-7695-9168

Sjoerd Hoogland: 0000-0002-3099-585X

Tapio Fabritius: 0000-0003-4729-8740

F. Pelayo García de Arquer: 0000-0003-2422-6234

Edward H. Sargent: 0000-0003-0396-6495

\section{Conflict of Interest}

The authors declare no conflict of interest. 


\section{Funding Sources}

This research was supported by the postdoctoral research fellow grant of the Academy of Finland (grant no. 296890). The authors also express their gratitude for the financial support received from the Academy of Finland's FIRI funding (grant no. 320017) and the University of Oulu Innovation Center PoC funding.

\section{ACKNOWLEDGMENTS}

R. Sliz and M. Lejay contributed equally to this work. The authors thank J. Lauri, E. Palmiano, D. Kopilovic, R. Wolowiec and A. Kam for their help and support over the course of this research.

\section{REFERENCES}

(1) García De Arquer, F. P.; Armin, A.; Meredith, P.; Sargent, E. H. Solution-Processed Semiconductors for Next-Generation Photodetectors. Nat. Rev. Mater. 2017, 2, 1-16.

(2) Kagan, C. R. Flexible Colloidal Nanocrystal Electronics. Chem. Soc. Rev. 2019, 48, 1626-1641.

(3) Haverinen, H. M.; Myllylä, R. A.; Jabbour, G. E. Inkjet Printing of Light Emitting Quantum Dots. Appl. Phys. Lett. 2009, 94, 94-97.

(4) Kim, L.; Anikeeva, P. O.; Coe-Sullivan, S. A.; Steckel, J. S.; Bawendi, M. G.; Bulović, V. Contact Printing of Quantum Dot Light-Emitting Devices. Nano Lett. 2008, 8, 4513-4517.

(5) Li, X.; Zhao, Y. B.; Fan, F.; Levina, L.; Liu, M.; Quintero-Bermudez, R.; Gong, X.; Quan, L. N.; Fan, J.; Yang, Z.; Hoogland, S.; Voznyy, O.; Lu, Z. H.; Sargent, E. H. Bright Colloidal Quantum Dot LightEmitting Diodes Enabled by Efficient Chlorination. Nat. Photonics 2018, 12, 159-164.

(6) Choi, J.-H.; Oh, S. J.; Paik, T.; Jo, P. S.; Zhao, T.; Murray, C. B.; Kagan, C. R.; Wang, H.; Sung, J.; Ye, X.; Diroll, B. T. Exploiting the Colloidal Nanocrystal Library to Construct Electronic Devices. Science (80-. ). 2016, 352, 205-208.

(7) Liu, M.; Voznyy, O.; Sabatini, R.; García De Arquer, F. P.; Munir, R.; Balawi, A. H.; Lan, X.; Fan, F.; Walters, G.; Kirmani, A. R.; Hoogland, S.; Laquai, F.; Amassian, A.; Sargent, E. H. Hybrid OrganicInorganic Inks Flatten the Energy Landscape in Colloidal Quantum Dot Solids. Nat. Mater. 2017, 16, 258-263.

(8) Xu, J.; Voznyy, O.; Liu, M.; Kirmani, A. R.; Walters, G.; Munir, R.; Abdelsamie, M.; Proppe, A. H.; Sarkar, A.; García De Arquer, F. P.; Wei, M.; Sun, B.; Liu, M.; Ouellette, O.; Quintero-Bermudez, R.; Li, 
J.; Fan, J.; Quan, L.; Todorovic, P.; Hairen T.; et al. 2D Matrix Engineering for Homogeneous Quantum Dot Coupling in Photovoltaic Solids. Nat. Nanotechnol. 2018, 13, 456-462.

(9) Clifford, J. P.; Konstantatos, G.; Johnston, K. W.; Hoogland, S.; Levina, L.; Sargent, E. H. Fast, Sensitive and Spectrally Tuneable Colloidal-Quantum-Dot Photodetectors. Nat. Nanotechnol. 2009, 4, 40-44.

(10) Kagan, C. R.; Lifshitz, E.; Sargent, E. H.; Talapin, D. V. Building Devices from Colloidal Quantum Dots. Science (80-. ). 2016, 353, aac5523.

(11) Fan, J. Z.; Liu, M.; Voznyy, O.; Sun, B.; Levina, L.; Quintero-Bermudez, R.; Liu, M.; Ouellette, O.; García De Arquer, F. P.; Hoogland, S.; Sargent, E. H. Halide Re-Shelled Quantum Dot Inks for Infrared Photovoltaics. ACS Appl. Mater. Interfaces 2017, 9, 37536-37541.

(12) Konstantatos, G.; Howard, I.; Fischer, A.; Hoogland, S.; Clifford, J.; Klem, E.; Levina, L.; Sargent, E. H. Ultrasensitive Solution-Cast Quantum Dot Photodetectors. Nature 2006, 442, 180-183.

(13) Wang, R.; Shang, Y.; Kanjanaboos, P.; Zhou, W.; Ning, Z.; Sargent, E. H. Colloidal Quantum Dot Ligand Engineering for High Performance Solar Cells. Energy Environ. Sci. 2016, 9, 1130-1143.

(14) Wei, J.; De Luna, P.; Bengio, Y.; Aspuru-Guzik, A.; Sargent, E. Use Machine Learning to Find Energy Materials. Nature 2017, 552, 23-25.

(15) Straus, D. B.; Goodwin, E. D.; Gaulding, E. A.; Muramoto, S.; Murray, C. B.; Kagan, C. R. Increased Carrier Mobility and Lifetime in CdSe Quantum Dot Thin Films through Surface Trap Passivation and Doping. J. Phys. Chem. Lett. 2015, 6, 4605-4609.

(16) Lauri, J.; Bykov, A. V.; Myllylä, R. Determination of Suspension Viscosity from the Flow Velocity Profile Measured by Doppler Optical Coherence Tomography. Photonics Lett. Pol. 2011, 3, 82-84.

(17) Zhao, T.; Goodwin, E. D.; Guo, J.; Wang, H.; Diroll, B. T.; Murray, C. B.; Kagan, C. R. Advanced Architecture for Colloidal PbS Quantum Dot Solar Cells Exploiting a CdSe Quantum Dot Buffer Layer. ACS Nano 2016, 10, 9267-9273.

(18) Konstantatos, G.; Sargent, E. H. Solution-Processed Quantum Dot Photodetectors. Proc. IEEE 2009, 97, $1666-1683$.

(19) Oh, S. J.; Straus, D. B.; Zhao, T.; Choi, J. H.; Lee, S. W.; Gaulding, E. A.; Murray, C. B.; Kagan, C. R. Engineering the Surface Chemistry of Lead Chalcogenide Nanocrystal Solids to Enhance Carrier Mobility and Lifetime in Optoelectronic Devices. Chem. Commun. 2017, 53, 728-731.

(20) Jo, J. W.; Kim, Y.; Choi, J.; de Arquer, F. P. G.; Walters, G.; Sun, B.; Ouellette, O.; Kim, J.; Proppe, A. H.; Quintero-Bermudez, R.; Fan, J.; Xu, J.; Tan, C. S.; Voznyy, O.; Sargent, E. H. Enhanced Open- 
Circuit Voltage in Colloidal Quantum Dot Photovoltaics via Reactivity-Controlled Solution-Phase Ligand Exchange. Adv. Mater. 2017, 29, 1-6.

(21) Ning, Z.; Dong, H.; Zhang, Q.; Voznyy, O.; Sargent, E. H. Solar Cells Based on Inks of N-Type Colloidal Quantum Dots. ACS Nano 2014, 8, 10321-10327.

(22) Derby, B. Inkjet Printing of Functional and Structural Materials: Fluid Property Requirements, Feature Stability, and Resolution. Annu. Rev. Mater. Res. 2010, 40, 395-414.

(23) Wijshoff, H. The Dynamics of the Piezo Inkjet Printhead Operation. Phys. Rep. 2010, 491, 77-177.

(24) Reis, N.; Derby, B. Experiments of Droplet Formation. Mater. Res. 2000, 625, 117-122.

(25) Jang, D.; Kim, D.; Moon, J. Influence of Fluid Physical Properties on Ink-Jet Printability. Langmuir 2009, 25, 2629-2635.

(26) Tai, J.; Gan, H. Y.; Liang, Y. N.; Lok, B. K. Control of Droplet Formation in Inkjet Printing Using Ohnesorge Number Category: Materials and Processes. 10th Electron. Packag. Technol. Conf. EPTC 2008, 761-766.

(27) Nallan, H. C.; Sadie, J. A.; Kitsomboonloha, R.; Volkman, S. K.; Subramanian, V. Systematic Design of Jettable Nanoparticle-Based Inkjet Inks: Rheology, Acoustics, and Jettability. Langmuir 2014, 30, $13470-13477$.

(28) De Iacovo, A.; Venettacci, C.; Colace, L.; Scopa, L.; Foglia, S. PbS Colloidal Quantum Dot Photodetectors Operating in the Near Infrared. Sci. Rep. 2016, 6, 1-9.

(29) Böberl, M.; Kovalenko, M. V.; Gamerith, S.; List, E. J. W.; Heiss, W. Inkjet-Printed Nanocrystal Photodetectors Operating up to $3 \mu \mathrm{m}$ Wavelengths. Adv. Mater. 2007, 19, 3574-3578.

(30) Tang, X.; Tang, X.; Lai, K. W. C. Scalable Fabrication of Infrared Detectors with Multispectral Photoresponse Based on Patterned Colloidal Quantum Dot Films. ACS Photonics 2016, 3, 2396-2404.

(31) Pal, B. N.; Robel, I.; Mohite, A.; Laocharoensuk, R.; Werder, D. J.; Klimov, V. I. High-Sensitivity p-n Junction Photodiodes Based on Pbs Nanocrystal Quantum Dots. Adv. Funct. Mater. 2012, 22, 17411748 .

(32) Manders, J. R.; Lai, T. H.; An, Y.; Xu, W.; Lee, J.; Kim, D. Y.; Bosman, G.; So, F. Low-Noise Multispectral Photodetectors Made from All Solution-Processed Inorganic Semiconductors. Adv. Funct. Mater. 2014, 24, 7205-7210.

(33) Masala, S.; Adinolfi, V.; Sun, J. P.; Gobbo, S. Del; Voznyy, O.; Kramer, I. J.; Hill, I. G.; Sargent, E. H. The Silicon: Colloidal Quantum Dot Heterojunction. Adv. Mater. 2015, 27, 7445-7450. 
(34) Kim, J. Y.; Adinolfi, V.; Sutherland, B. R.; Voznyy, O.; Kwon, S. J.; Kim, T. W.; Kim, J.; Ihee, H.; Kemp, K.; Adachi, M.; Yuan, M.; Kramer, I.; Zhitomirsky, D.; Hoogland, S.; Sargent, E. H. Single-Step Fabrication of Quantum Funnels via Centrifugal Colloidal Casting of Nanoparticle Films. Nat. Commun. 2015, 6, 1-9.

(35) Torrisi, F.; Hasan, T.; Wu, W.; Sun, Z.; Lombardo, A.; Kulmala, T. S.; Hsieh, G. W.; Jung, S.; Bonaccorso, F.; Paul, P. J.; Chu, D.; Ferrari, A. C. Inkjet-Printed Graphene Electronics. ACS Nano 2012, 6, 2992-3006.

(36) Chuang, C. H. M.; Brown, P. R.; Bulović, V.; Bawendi, M. G. Improved Performance and Stability in Quantum Dot Solar Cells through Band Alignment Engineering. Nat. Mater. 2014, 13, 796-801.

Zhang, M.; Zhu, L.; Hou, Y.; Hu, Y.; Wang, Y.; Teng, F.; Zhang, F.; Lou, Z. High-Performance Photodiode-Type Photodetectors Based on Polycrystalline Formamidinium Lead Iodide Perovskite Thin Films. Sci. Rep. 2018, 8, 1-9.

(38) Hawash, Z.; Ono, L. K.; Qi, Y. Recent Advances in Spiro-MeOTAD Hole Transport Material and Its Applications in Organic-Inorganic Halide Perovskite Solar Cells. Adv. Mater. Interfaces 2018, 5, 1-22.

(39) Xu, X.; Feng, L.; He, S.; Jin, Y.; Guo, X. Solution-Processed Zinc Oxide Thin-Film Transistors with a Low-Temperature Polymer Passivation Layer. IEEE Electron Device Lett. 2012, 33, 1420-1422.

(40) Snaith, H. J.; Grätzel, M. Enhanced Charge Mobility in a Molecular Hole Transporter via Addition of Redox Inactive Ionic Dopant: Implication to Dye-Sensitized Solar Cells. Appl. Phys. Lett. 2006, 89, 262114.

(41) Boles, M. A.; Ling, D.; Hyeon, T.; Talapin, D. V. The Surface Science of Nanocrystals. Nat. Mater. 2016, 15, 141-153.

(42) Ning, Z.; Zhitomirsky, D.; Adinolfi, V.; Sutherland, B.; Xu, J.; Voznyy, O.; Maraghechi, P.; Lan, X.; Hoogland, S.; Ren, Y.; Sargent, E. H. Graded Doping for Enhanced Colloidal Quantum Dot Photovoltaics. Adv. Mater. 2013, 25, 1719-1723.

(43) Tan, H.; Jain, A.; Voznyy, O.; Lan, X.; De Arquer, F. P. G.; Fan, J. Z.; Quintero-Bermudez, R.; Yuan, M.; Zhang, B.; Zhao, Y.; Fan, F.; Li, P.; Quan, L. N.; Zhao, Y.; Lu, Z. H.; Yang, Z.; Hoogland, S.; Sargent, E. H. Efficient and Stable Solution-Processed Planar Perovskite Solar Cells via Contact Passivation. Science (80-. ). 2017, 355, 722-726. 
For Table of Contents Only

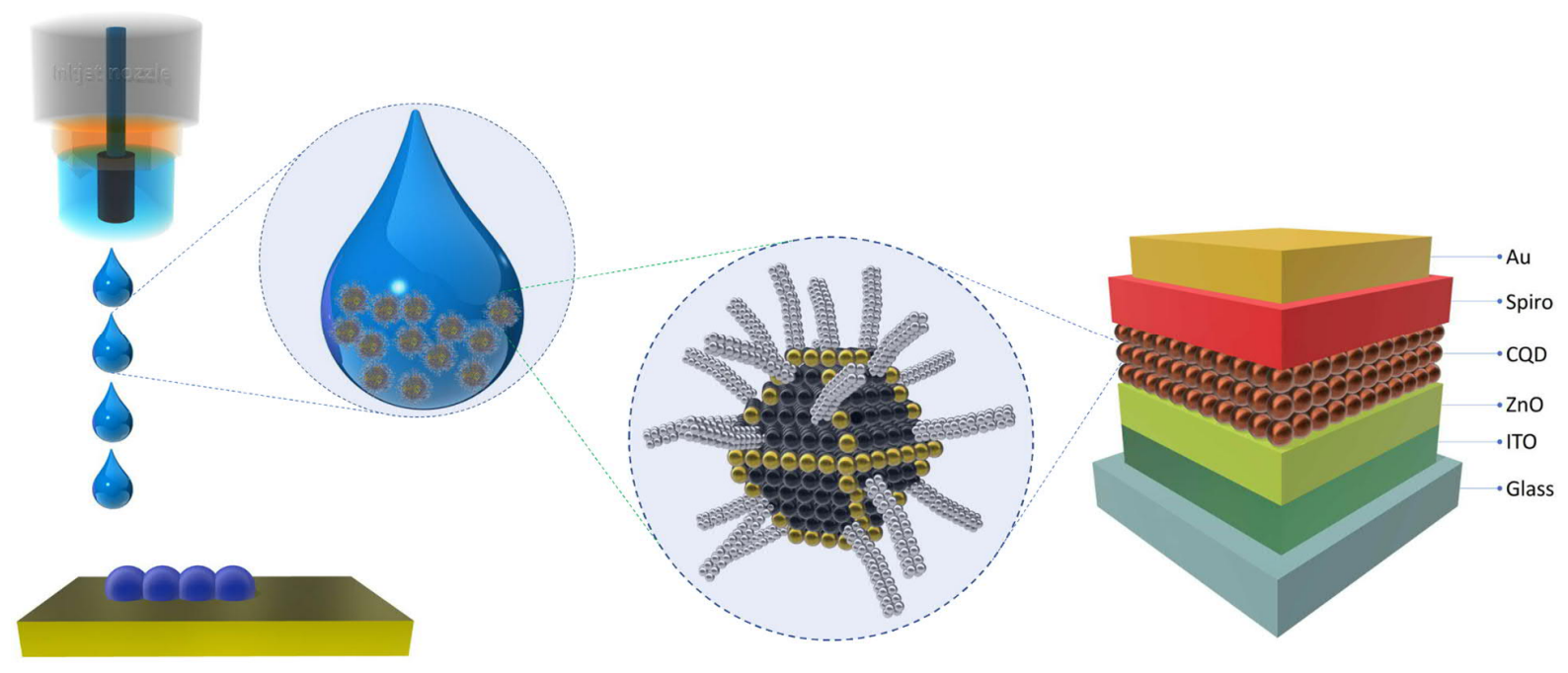

